\title{
Prognostic value of programmed cell death ligand-1 expression in ovarian cancer: an updated meta-analysis
}

\author{
Jinlan Piao, MD', Hyun Ji Lim, MD², Maria Lee, MD, PhD ${ }^{1,2}$ \\ Department of Obstetrics and Gynecology, ${ }^{1}$ Seoul National University College of Medicine, ${ }^{2}$ Seoul National University Hospital, Seoul, Korea
}

\section{Objective}

To investigate the prognostic significance of programmed cell death ligand-1 (PD-L1) in ovarian cancer.

\section{Methods}

PubMed, Embase, and Cochrane Library databases were searched to identify studies that examined the prognostic significance of immunohistochemically assessed PD-L1 expression in histologically confirmed ovarian cancer. Eleven studies on PD-L1 expression involving 1,296 patients with ovarian cancer were included in this meta-analysis. Pooled hazard ratios (HRs) with corresponding 95\% confidence intervals ( $\mathrm{Cls}$ ) were analyzed. Relationship between PDL1 expression, and overall survival (OS) or progression-free survival (PFS) among patients with ovarian cancer was assessed. Subgroup analysis was performed based on the race, histologic type, and tumor International Federation of Gynecology and Obstetrics stage to evaluate the source of heterogeneity. Begg's Funnel plot and Egger's linear test were used to evaluate publication bias. Random-effects model was implemented when significant between-study heterogeneity $\left(I^{2}>50 \%\right)$ was observed.

Results

We found no correlation between PD-L1 expression, and OS (HR, 1.13; 95\% Cl, 0.95-1.36; I' $=78 \%)$ or PFS (HR, 1.07; $\left.95 \% \mathrm{Cl}, 0.88-1.30 ; I^{2}=75 \%\right)$ in ovarian cancer. Subgroup analyses showed that higher PD-L1 expression was associated with poor OS in non-Asian patients with ovarian cancer $\left(\mathrm{HR}, 1.26 ; 95 \% \mathrm{Cl}, 1.07-1.481 ; I^{2}=59 \%\right)$. We found that upregulated PD-L1 expression to be a positive predictor for OS in serous ovarian cancer (HR, 0.98; $95 \% \mathrm{Cl}, 0.76-$ $\left.1.26 ; I^{2}=74 \%\right)$ and a negative predictor for OS in non-serous ovarian cancer (HR, $\left.1.29 ; 95 \% \mathrm{Cl}, 1.03-1.61 ; \mathrm{I}^{2}=64 \%\right)$ Furthermore, high PD-L1 expression was found to be a negative predictor for PFS of patients with non-serous ovarian cancer (HR, 1.12; 95\% Cl, 0.96-1.29; $\left.I^{2}=37 \%\right)$.

\section{Conclusion}

Our meta-analysis suggests that PD-L1 expression is not associated with patient risk for ovarian cancer.

Keywords: Ovarian cancer; PD-L1; Survival rate; Progression-free survival; Prognosis

\section{Introduction}

Ovarian cancer is reported as the sixth most common cancer and seventh most leading cause of cancer-associated death among women worldwide [1]. Additionally, ovarian cancer is known to be the second most common gynecological cancer and leading cause of gynecological cancer-related death in the western world [1]. Majority of the patients with ovarian cancer are initially diagnosed at an advanced stage [International Federation of Gynecology and Obstetrics (FIGO) stage III] due to the lack of clinical appearance. Treatment options for these patients continue to be surgery and platinum-based
Received: 2019.08.02. Revised: 2019.11.12. Accepted: 2020.01.16. Corresponding author: Maria Lee, $\mathrm{MD}, \mathrm{PhD}$

Department of Obstetrics and Gynecology, Seoul National University College of Medicine, 101 Daehak-ro, Jongno-gu, Seoul 03080, Korea

E-mail: marialeemd@gmail.com

https://orcid.org/0000-0002-8017-3176

Articles published in Obstet Gynecol Sci are open-access, distributed under the terms of the Creative Commons Attribution Non-Commercial License (http://creativecommons. org/licenses/by-nc/3.0/) which permits unrestricted non-commercial use, distribution, and reproduction in any medium, provided the original work is properly cited.

Copyright $\odot 2020$ Korean Society of Obstetrics and Gynecology 


\section{Obstetrics \& Gynecology Science}

Jinlan Piao, et al. Prognostic value of PD-L1

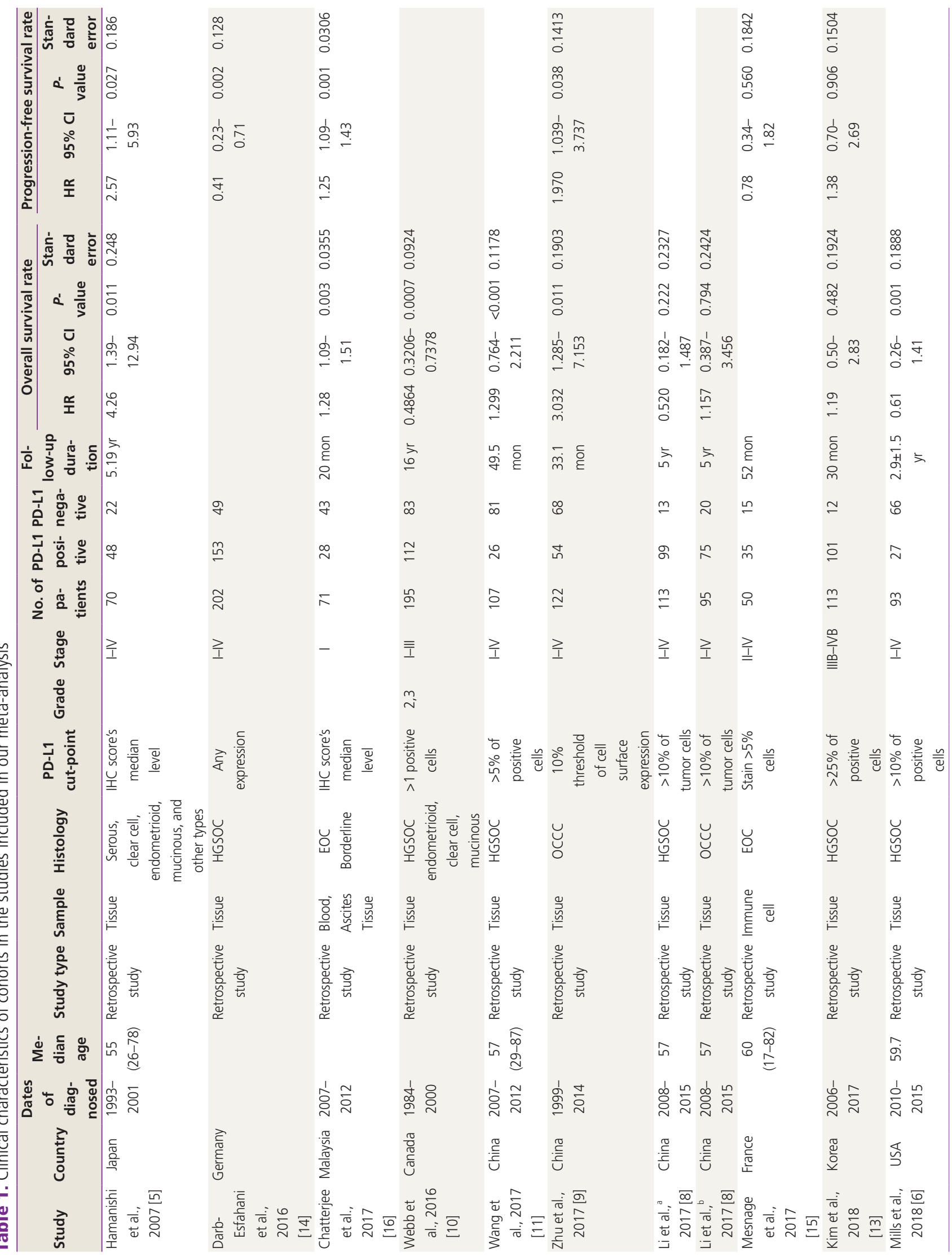




\section{Obstetrics \& Gynecology Science}

Vol. 63, No. 3, 2020

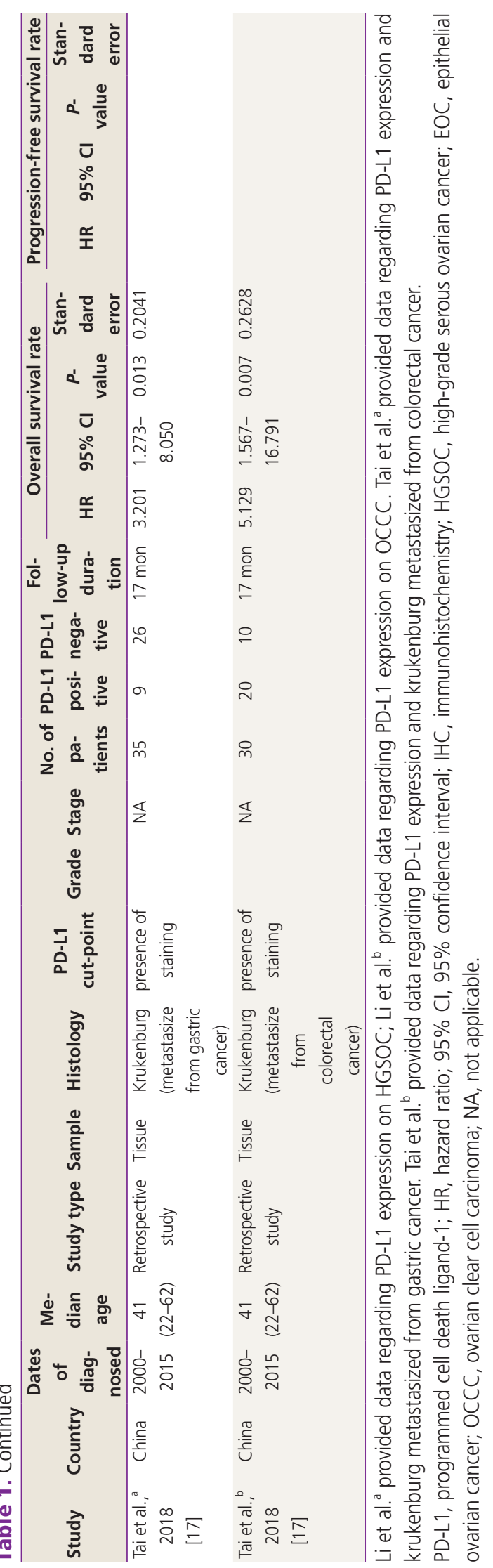

cytotoxic chemotherapy with five-year survival rates of less than 20\% [2].

Programmed cell death 1 (PD-1) receptor belongs to the B7-CD28 family of costimulatory receptors. PD-1 is expressed on the surface of T, B, and Natural Killer (NK) cells and has been shown to play key roles in their activation and apoptosis. Programmed cell death ligand-1 (PD-L1) is one of the ligands of PD-1 that is expressed on both tumor and immune cells such as dendritic cells (DCs) or macrophages. Typically, neoantigens produced by tumor cells are recognized by DCs, which further activate cytotoxic T cells. Activated T cells then infiltrate the tumor environment, bind to cancer cells, and release cytokines that trigger apoptosis in target cancer cells. To escape such anti-tumor mechanism, tumor cells have been observed to often overexpress PD-L1 that binds to PD-1 receptors on the activated $T$ cells, thereby inhibiting cytotoxic T cells. Thus, PD-L1 has been known to be part of a crucial immunological escape mechanism, which promotes tumor cell growth, proliferation, and metastasis [3,4]. However, there are conflicting reports on the relationship between PDL1 expression and prognosis in ovarian cancer. For instance, Hamanishi et al. [5] reported that PD-L1 expression was associated with poor prognosis in ovarian cancer; however, no relationship between PD-L1 expression and prognosis of ovarian cancer was observed by Mills et al. [6]. Recently, a metaanalysis on PD-L1 expression and prognosis in ovarian cancer has been published in 2018 [7]. This meta-analysis found that PD-L1 expression may be a negative predictor for prognosis of patients with ovarian cancer from Asian countries, while a promising positive predictor for non-Asian patients with ovarian cancer. To gain further clarity on the relationship between PD-L1 expression and ovarian cancer progression, we conducted the present meta-analysis after contemplating high-quality articles that have been recently published.

\section{Materials and methods}

\section{Literature search}

Two authors performed literature search using PubMed, EMBASE, and Cochrane Library databases. Literature search was performed using the following key words: "programmed death ligand 1" or "PD-L1" or "PDL1" or "B7-H1" or "B7H1" or "CD274," and "ovarian" or "ovary," and "cancer" or "carcinoma" or "neoplasm" or "tumour." In addition, a 


\section{Obstetrics \& Gynecology Science}

Jinlan Piao, et al. Prognostic value of PD-L1

manual search from reference lists of all the eligible studies was conducted to obtain additional references. The final search was conducted on November 7, 2019. Overall, 838 articles were searched using the key words, and 265 articles were excluded due to duplication. Further, 507 articles were excluded because the title and abstract were not relevant to PD-L1 expression and ovarian cancer. Additionally, 55 articles were excluded as sufficient data on overall survival (OS) or progression-free survival (PFS) rate were not available. Finally, 11 articles were included in this meta-analysis. Ethical approval or patient consent was not required for our metaanalysis as only previously published studies were analyzed.

\section{Eligibility criteria}

The inclusion criteria for the studies in our meta-analysis were as follows: studies focusing on ovarian cancer, involving patients with histologically confirmed ovarian cancer, studies on PD-L1 (B7-H1) expression that was detected by immunohistochemistry $(\mathrm{IHC})$ staining of tumor tissue, and studies investigating potential association between PD-L1 expression and OS or PFS of patients with ovarian cancer. IHC scoring system for PD-L1 was developed based on percentage of positive tumor cells and staining intensity. Staining intensity was graded as 0 (no staining), 1 (weak), 2 (intermediate), or 3 (strong), whereas distribution of the cellular staining was graded as 0 (none), 1 (<10\% of cells), 2 (11-50\% of cells), 3 ( $51-75 \%$ of cells), or $4(>75 \%$ of cells). Scores 0 and 1 were defined as low expression, and scores 2 and 3 were defined as high expression [5-8], and the PD-L1 cut-off point was different in each article (Table 1). All articles included in this meta-analysis were written in English.

\section{Data extraction}

Two authors, Maria Lee and Jinlan Piao, independently extracted the following information from every study included in our meta-analysis: name of the first author, publication year, country, median age, study type, source of resected tumor histology, FIGO stage and grade, number of patients, number of PD-L1-positive and negative patients, hazard ratios (HRs) with corresponding 95\% confidence intervals ( $\mathrm{Cls}$ ), OS, and PFS. The end point for PFS was defined as the day of recurrence, while that for OS was defined as the day when the patient was confirmed alive or dead, respectively [9].

\section{Assessment of study quality}

Two authors independently assessed the quality of the in-

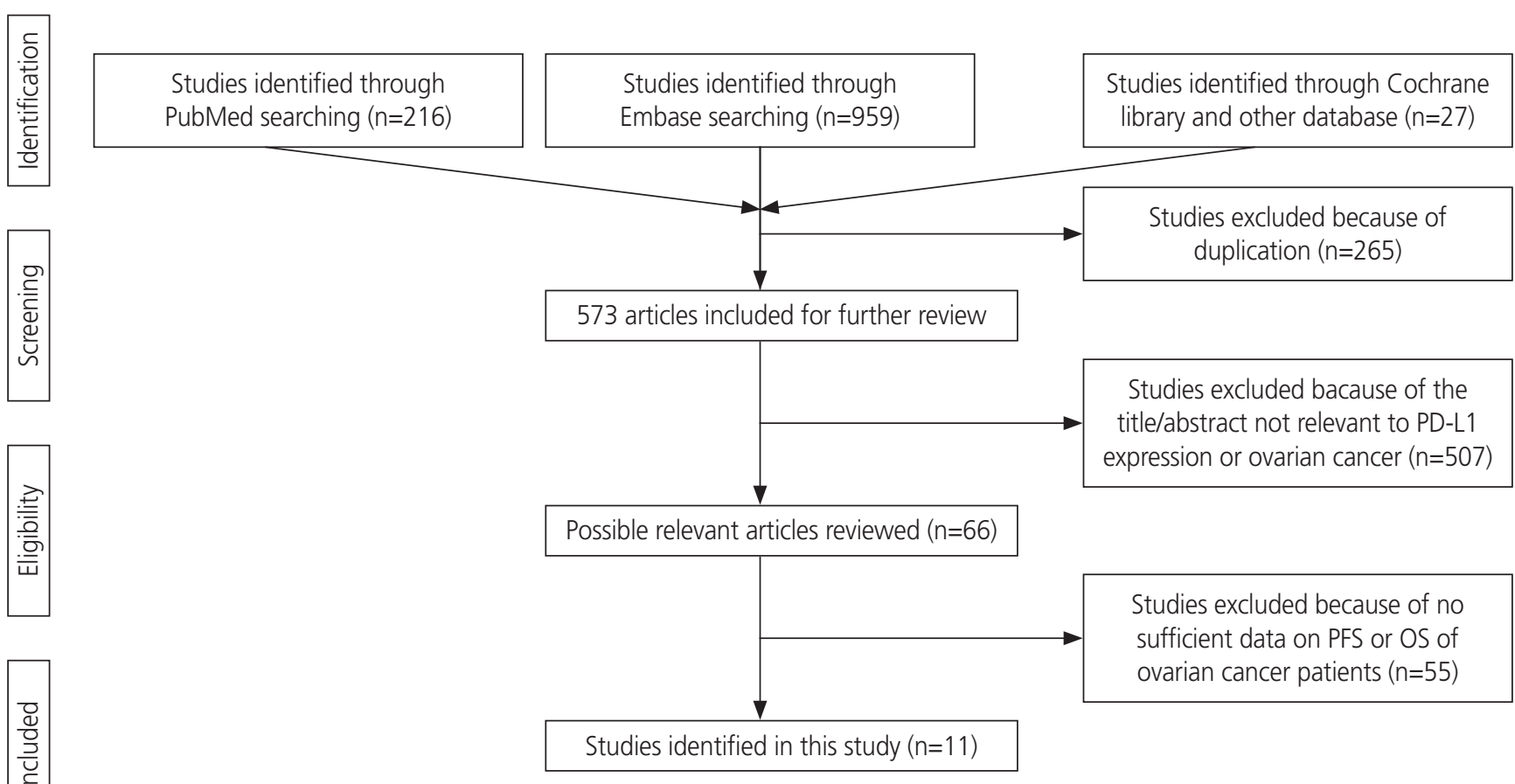

Fig. 1. Flow chart of literature search and study design. 


\section{Obstetrics \& Gynecology Science}

Vol. 63 , No. 3, 2020

cluded studies using Newcastle-Ottawa Quality Assessment Scale (NOS). Disagreements in scoring were resolved by discussing with a third reviewer. NOS was used to evaluate the following three parameters: selection (0-4 points), comparability (0-2 points), and outcome (0-3 points). The highest NOS score was 9 points, and studies scoring greater than 5 were classified as high-quality.

\section{Statistical methods}

Pooled HRs with corresponding 95\% Cls were used to evaluate the association between PD-L1 expression and prognosis of patients with ovarian cancer. Subgroup analysis was performed based on the race, histologic type of ovarian cancer, and tumor FIGO stage of the enrolled patients with ovarian cancer. Random-effects model was implemented when significant heterogeneity $\left(I^{2}>50 \%\right.$ or $\left.P<0.1\right)$ was detected between the studies. Potential publication bias was visually assessed by Begg's funnel plot and Egger's linear test. This meta-analysis was performed using Review Manager 5.3 and STATA 15. $P<0.05$ was considered to be statistically significant.

\section{Results}

\section{Search results}

A total of 838 articles were screened for this study. After exempting duplicates, the title and abstract were screened for relevance. Articles that were not directly related to the subject of our meta-analysis or that did not provide a potential relationship between PD-L1 expression data and OS or PFS of patients with ovarian cancer were excluded. Finally, 11 articles (13 comparisons) published between 2006 and 2018 with NOS $\geq 5$ were included in our meta-analysis. The screening process is described in Fig. 1.

\section{Study characteristics}

Characteristics of the included studies are shown in Table 1. In our meta-analysis, 11 studies (13 comparisons) were included, which involved 1,296 patients with ovarian cancer that were examined for PD-L1 expression and prognosis. The included studies were typically high-quality with NOS $\geq 5$ (Table 2). In all the selected studies, PD-L1 expression levels were detected by IHC staining. The included studies were observed to have 7 patient cohorts with high-grade serous ovarian cancer (HGSOC) [8,10-14], 4 cohorts with ovarian

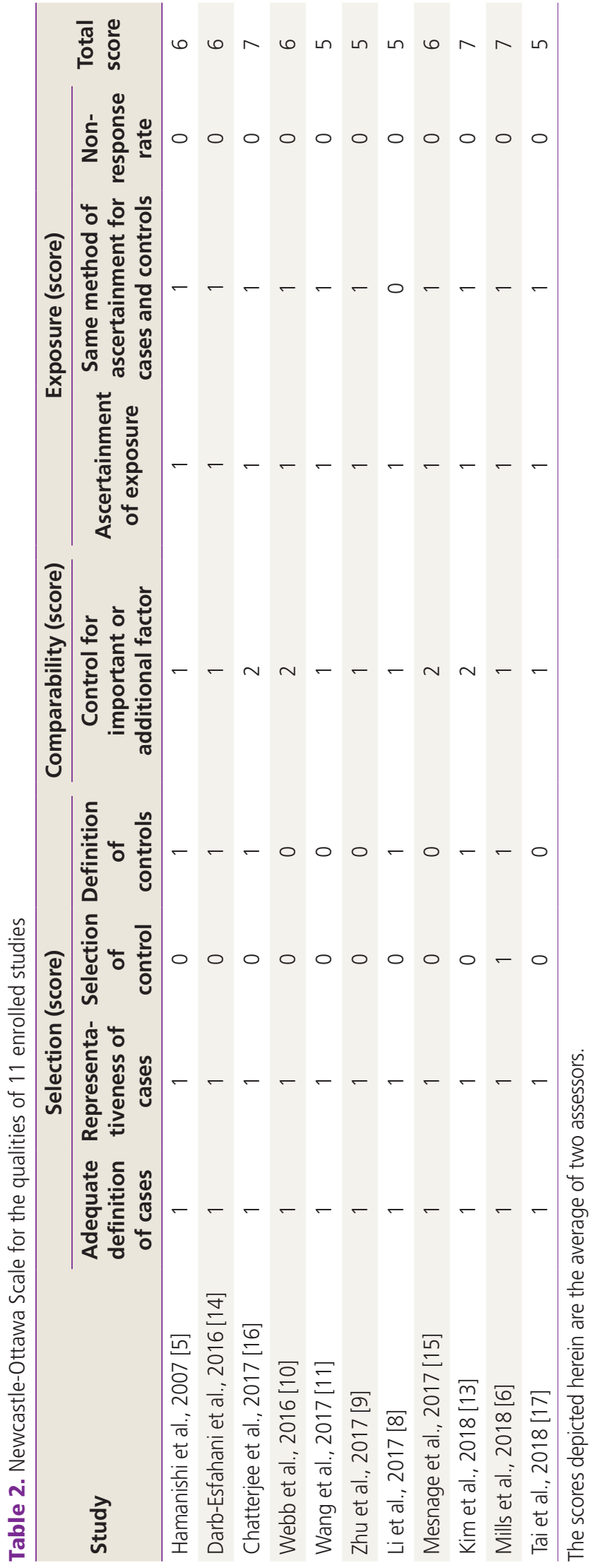




\section{Obstetrics \& Gynecology Science}

Jinlan Piao, et al. Prognostic value of PD-L1

clear cell carcinoma (OCCC) [5,8,9], 2 cohorts with endometrioid ovarian cancer $[5,10], 2$ cohorts with mucinous ovarian cancer $[5,10], 2$ cohorts with epithelial ovarian cancer (EOC) [15,16], 1 cohort with borderline ovarian cancer [16], and 2 cohorts with krukenburg tumor of ovarian cancer [17]. Among the 1,296 patients included in these 11 studies, 640 patients were found to be diagnosed with early stage (FIGO stage I-II) ovarian cancer and 519 patients with advanced stage (FIGO stage III-IV) ovarian cancer (Table 1).

\section{Association between PD-L1 expression and OS or PFS in ovarian cancer}

Among the 13 potential comparisons, 11 assessments were observed to analyze the relationship between PD-L1 expression and OS of patients with ovarian cancer. The data were pooled using random-effects model due to significant heterogeneity between the studies $\left(I^{2}=78 \%, P<0.0001\right)$. Based on the pooled HR, we found that increase in PD-L1 expression was not significantly associated with OS of patients with ovarian cancer $(\mathrm{HR}, 1.13 ; 95 \% \mathrm{Cl}, 0.96-1.36 ; P<0.001$; $\mathrm{I}^{2}=78 \%$ ) (Figs. 2 and 3). Among the 11 included studies, 6 evaluated PD-L1 expression and PFS of patients with ovarian cancer. The data were pooled using random-effects model due to significant heterogeneity between the studies $\left(I^{2}=78 \% ; P<0.001\right)$. Our results indicated that increase in PDL1 expression was not significantly associated with PFS of
$H R$

$\mathrm{HR}$

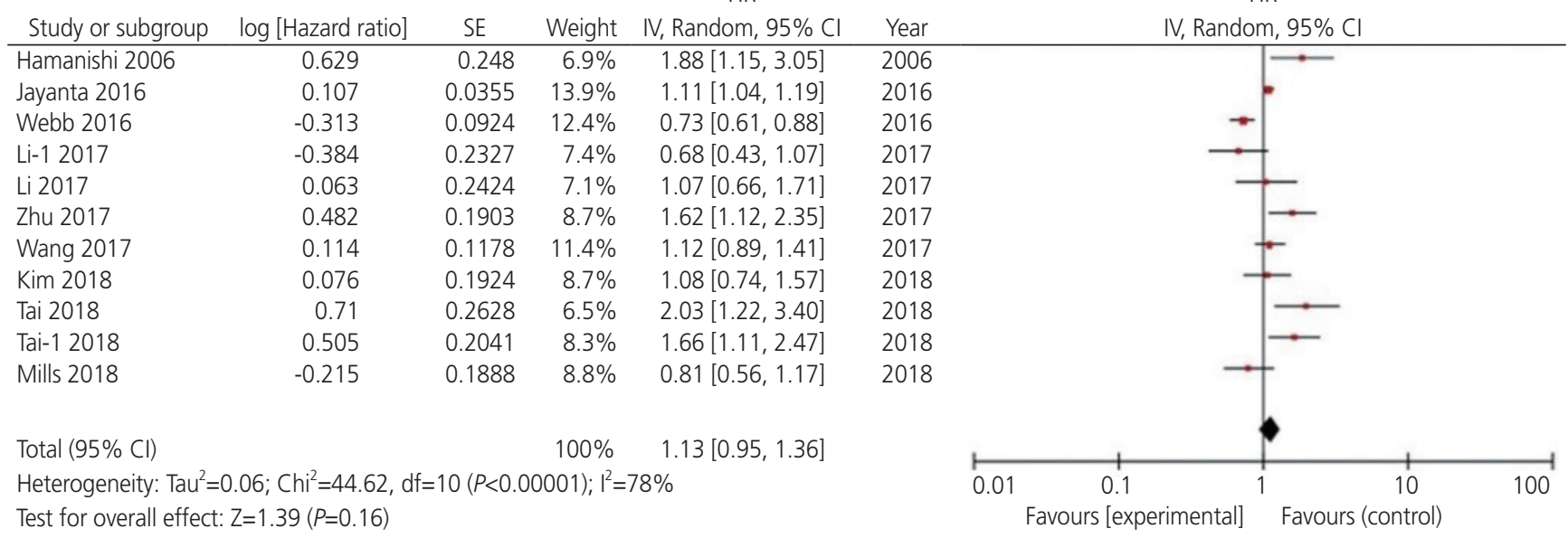

Fig. 2. Forest plot for overall survival rate of patients with ovarian cancer. HR, hazard ratio; SE, standard error; IV, inverse variance random; $95 \% \mathrm{Cl}, 95 \%$ confidence interval.
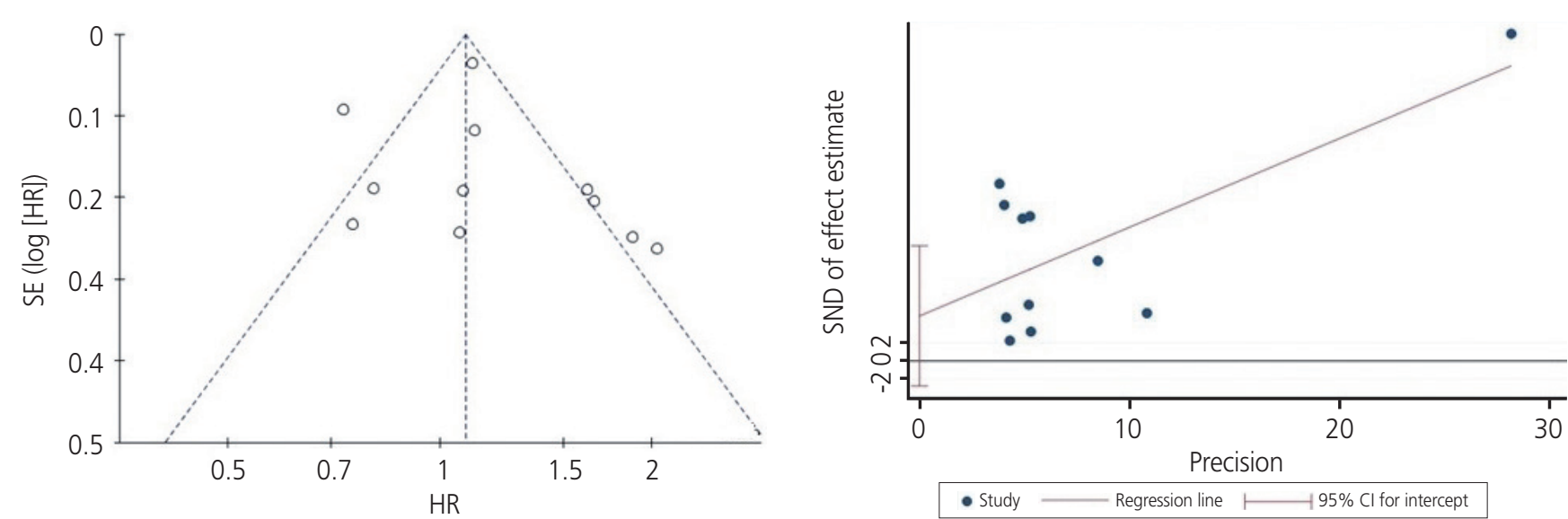

Fig. 3. Begg's funnel plot $(P=0.029)$ and Egger's linear regression test $(P=0.180)$ for assessing potential publication bias while establishing the potential relationship between programmed cell death ligand-1 expression and overall survival rate in patients with ovarian cancer. $\mathrm{SE}$, standard error; HR, hazard ratio; SND, standard normal deviate; 95\% Cl, 95\% confidence interval. 


\section{Obstetrics \& Gynecology Science}

Vol. 63, No. 3, 2020

patients with ovarian cancer ( $\mathrm{HR}, 1.07 ; 95 \% \mathrm{Cl}, 0.88-1.30$; $I^{2}=75 \% ; P=0.51$ ) (Fig. 4).

Subgroup analyses showed that higher PD-L1 expression was associated with poor OS of non-Asian patients with ovarian cancer (HR, 1.26; $\left.95 \% \mathrm{Cl}, 1.07-1.481 ; \mathrm{I}^{2}=59 \%\right)$; however, a similar trend was not observed in Asian patients. Upregulated PD-L1 expression was found to be a positive predictor for OS in serous ovarian cancer (HR, 0.98; $95 \%$ $\mathrm{Cl}, 0.76-1.26 ; \mathrm{I}^{2}=74 \%$ ) and a negative predictor for OS in non-serous ovarian cancer (HR, 1.29; $95 \% \mathrm{Cl}, 1.03-1.61$; $\left.\mathrm{I}^{2}=64 \%\right)$. In contrast, higher expression of PD-L1 was observed to be a negative predictor for early stage (HR, 1.20; $\left.95 \% \mathrm{Cl}, 0.86-1.68 ; I^{2}=90 \%\right)$ and advanced stage ovarian cancer ( $\left.\mathrm{HR}, 1.09 ; 95 \% \mathrm{Cl}, 0.90-1.33 ; \mathrm{I}^{2}=54 \%\right)$. Additionally, we found that PD-L1 expression was a negative predictor for PFS of patients with serous (HR, 1.04; $95 \% \mathrm{Cl}, 0.65-1.67$;
$\left.\mathrm{I}^{2}=86 \%\right)$ and non-serous ovarian cancer ( $\mathrm{HR}, 1.12 ; 95 \% \mathrm{Cl}$, $0.96-1.29 ; I^{2}=37 \%$ ) (Tables 3 and 4).

\section{Publication bias}

Funnel plots were used to assess the studies included in our meta-analysis for potential publication bias. As shown in Figs. 3 and 5, funnel plots revealed asymmetry for OS (Begg's funnel plot, $P=0.029$ ) but did not show asymmetry for the PFS (Begg's funnel plot, $P=0.612$ ) of patients with ovarian cancer. Egger's linear tests were used to identify publication bias in OS (Egger's test, $P=0.180$ ).

\section{Discussion}

PD-1 is a member of the CD28 costimulatory receptor super-

$H R$

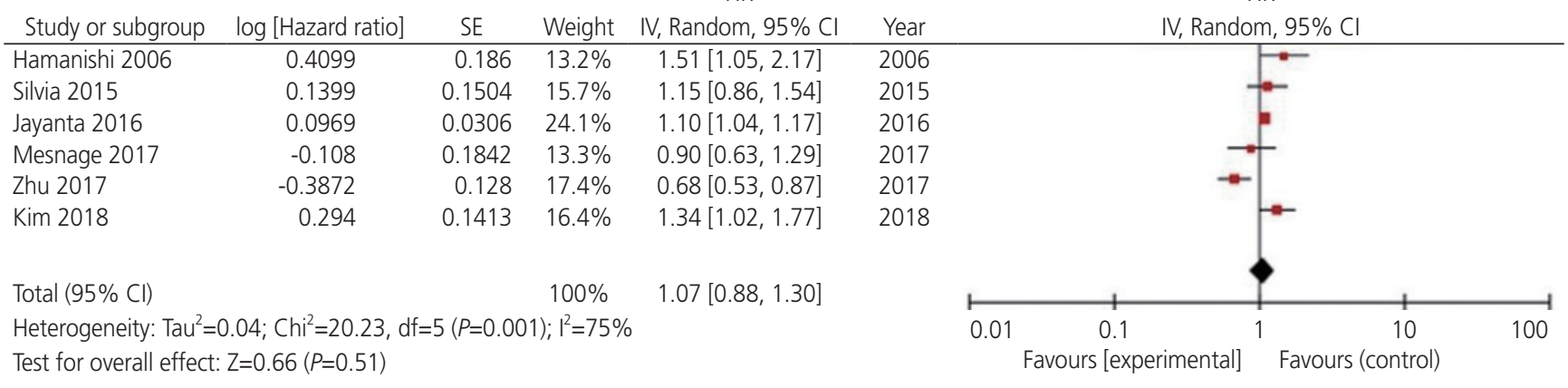

Fig. 4. Forest plot for determining progression-free survival rate of patients with ovarian cancer. HR, hazard ratio; SE, standard error; IV, inverse variance random; $95 \% \mathrm{Cl}, 95 \%$ confidence interval.

Table 3. Subgroup analysis for programmed cell death ligand-1 expression and the outcome of overall survival rate of patients with ovarian cancer

\begin{tabular}{|c|c|c|c|c|c|}
\hline \multirow{2}{*}{ Subgroup } & \multirow{2}{*}{ No. of comparisons } & \multirow{2}{*}{ Relative HR } & \multicolumn{3}{|c|}{ Pooled HRs } \\
\hline & & & HR (95\% Cls) & $P$-value & $I^{2}$ \\
\hline \multicolumn{6}{|l|}{ Race } \\
\hline Asian & 9 & 1 & $0.77(0.66-0.89)$ & 0.590 & $0 \%$ \\
\hline Non-Asian & 4 & 1 & $1.26(1.07-1.48)$ & 0.010 & $59 \%$ \\
\hline \multicolumn{6}{|l|}{ Histology } \\
\hline Serous & 7 & 1 & $0.98(0.76-1.26)$ & 0.002 & $74 \%$ \\
\hline Non-serous & 6 & 1 & $1.29(1.03-1.61)$ & 0.020 & $64 \%$ \\
\hline \multicolumn{6}{|l|}{ Stage } \\
\hline Early stage & 4 & 1 & $1.20(0.86-1.68)$ & $<0.001$ & $90 \%$ \\
\hline Advanced stage & 9 & 1 & $1.09(0.90-1.33)$ & 0.030 & $54 \%$ \\
\hline
\end{tabular}

HR, hazard ratio; 95\% Cl, 95\% confidence interval. 


\section{Obstetrics \& Gynecology Science}

Jinlan Piao, et al. Prognostic value of PD-L1

Table 4. Subgroup analysis for PD-L1 expression and the outcome of progression-survival rate of patients with ovarian cancer

\begin{tabular}{|c|c|c|c|c|c|}
\hline \multirow{2}{*}{ Subgroup } & \multirow{2}{*}{ No. of comparisons } & \multirow{2}{*}{ Relative HR } & \multicolumn{3}{|c|}{ Pooled HRs } \\
\hline & & & HR (95\% Cls) & $P$-value & $I^{2}$ \\
\hline \multicolumn{6}{|l|}{ Race } \\
\hline Asian & 4 & 1 & $1.18(1.04-1.35)$ & 0.21 & $33 \%$ \\
\hline Non-Asian & 2 & 1 & $0.76(0.58-0.99)$ & 0.21 & $35 \%$ \\
\hline \multicolumn{6}{|l|}{ Histology } \\
\hline Serous & 3 & 1 & $1.04(0.65-1.67)$ & 0.001 & $86 \%$ \\
\hline Non-serous & 3 & 1 & $1.12(0.96-1.29)$ & 0.02 & $37 \%$ \\
\hline \multicolumn{6}{|l|}{ Stage } \\
\hline Early stage & 1 & 1 & $1.10(1.04-1.17)$ & - & - \\
\hline Advanced stage & 5 & 1 & $1.06(0.79-1.44)$ & 0.001 & $79 \%$ \\
\hline
\end{tabular}

HR, hazard ratio; 95\% Cl, 95\% confidence interval.

Funnel plot with 95\% confidence limits

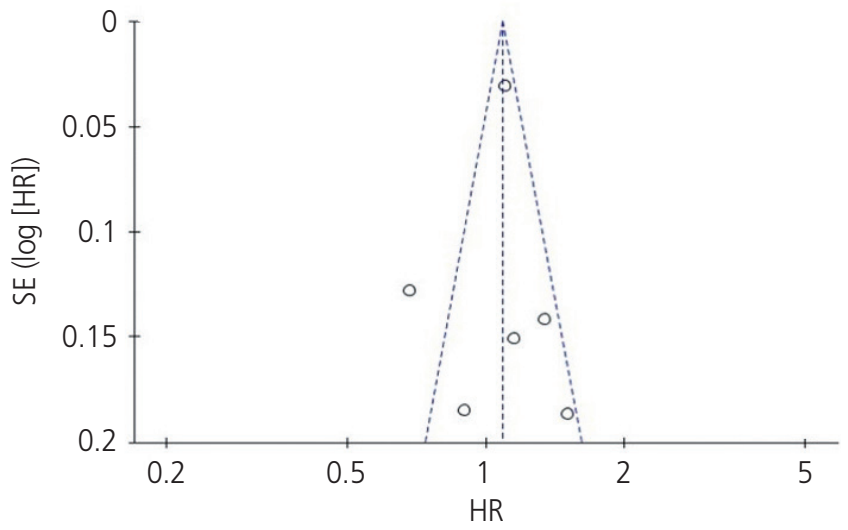

Fig. 5. Begg's funnel plot test $(P=0.612)$ for assessing potential publication bias while establishing the potential relationship between programmed cell death ligand-1 expression and progression-free survival rate in patients with ovarian cancer. SE, standard error; HR, hazard ratio.

family. PD-1 transmits inhibitory signals that abrogate $\mathrm{T}$ cell receptor-mediated activating signals, thereby preventing further antigen-mediated activation of T-cells. One of its ligands, known as PD-L1 or CD274, is mainly expressed on the surface of tumor cells and antigen-presenting cells in many solid malignancies [18,19]. In addition to tumor cells, high expression levels of PD-L1 have been observed in human tumorassociated antigen-presenting cells, which includes tumor environment-associated DCs, tumor-draining lymph node DCs, macrophages, fibroblasts, and T cells [19-21]. PD-L1 is an important immune regulatory factor, and as a receptor for PD-1, it plays a key role in the immune escape mechanism of cancer cells. PD-L1 specifically binds to the PD-1 receptor of T cells and impairs the activation and differentiation of $T$ cells. Tumor-infiltrating immune cells have been shown to induce cytokines such as interferons and vascular endothelial growth factors that upregulate PD-L1 expression [22-25]. PD-L1 upregulation is known to regulate various intracellular signaling pathways both at the transcriptional and translational levels. Additionally, this upregulation has been reported to alter the production of various pre-inflammatory factors and cytokines that are secreted in the tumor microenvironment $[26,27]$. Therefore, PD-L1 overexpression may potentially influence cancer progression and be associated with poor prognoses. Studies on the expression, regulation, and function of the PD pathway in human cancer microenvironment have provided scientific rationales that have directly supported the current clinical application for blocking the PD pathway $[18,20]$. Moreover, PD-L1 is known to be expressed and associated with the prognosis of many human cancers such as lung [28], breast [29], hepatocellular [30], cervical [31], and ovarian cancers $[8,23,32]$.

Since PD-1/PD-L1 pathway is suggested to play a pivotal role in the immune escape mechanism and growth of cancer cells $[4,33]$, the relationship between PD-L1 expression and prognosis of ovarian cancer has markedly attracted several researchers and clinicians. Previous reports [34-36] have suggested the crucial role of PD-1/PD-L1 inhibition in ovarian cancer treatment. Furthermore, some studies have proposed that PD-L1 may not only be a good therapeutic target but also a prognostic biomarker for ovarian cancer $[5,9-11,15,17]$. However, other studies have yielded discrepant results. Thus, 


\section{Obstetrics \& Gynecology Science}

Vol. 63, No. 3, 2020

the issue still remains controversial concerning ovarian cancer. A previous meta-analysis that investigated the association between PD-L1 expression and prognosis of ovarian carcinoma found that PD-L1 expression is a poor-prognosis biomarker in Asian populations; however, it is a good-prognosis biomarker in non-Asian patients with ovarian cancer [7].

In this meta-analysis, we included 11 studies (13 comparisons). From the selected studies, 11 comparisons were found analyzing the relationship between PD-L1 expression and OS of patients with ovarian cancer, while 6 comparisons were found analyzing the relationship between PD-L1 expression and PFS in ovarian cancer. Overall, our meta-analysis showed that PD-L1 expression was not associated with OS or PFS of patients with ovarian cancer. As high heterogeneity was observed among the included studies, we performed subgroup analysis to investigate the source of heterogeneity. We found that in Asian patients, PD-L1 expression was not associated with OS in ovarian cancer (HR, 0.77; 95\% Cl, 0.66-0.89; $\left.P=0.59 ; I^{2}=0 \%\right)$. However, in non-Asian patients with ovarian cancer, high PD-L1 expression was observed to increase the risk of OS (HR, 1.26; 95\% Cl, 1.07-1.48; $\left.P=0.01 ; I^{2}=59 \%\right)$. Intriguingly, our data differed from the conclusions of the previous meta-analysis published in 2018 [7]. Furthermore, our meta-analysis revealed that PD-L1 expression was associated with histology, tumor stage in OS of patients with ovarian cancer. In clinical studies, PD-L1 expression in cancer was mostly studied at the protein level using IHC. However, there are limitations in PD-L1 IHC standardization that may end up with discordant results. While reviewing the included articles, we found that the PD-L1 cut-off point was different in each study. Thus, it is possible that the variations in the cut-off value and antibodies of PD-L1 might have caused the heterogeneity. Moreover, the previous studies revealed that patients with IHC-positive tumors may not respond to treatment [37].

Our meta-analysis had certain limitations. For example, at the literature review level, we cannot exclude the possibility that despite our best efforts, some relevant studies may have been inadvertently excluded or not found in our queried databases using the selected search terms. Moreover, only studies published in English language journals were included in our meta-analysis, which introduces a selection bias. We are aware of the fact that although our qualitative assessment of the primary studies would have revealed certain inherent biases, it would have not encapsulated the aggregate effect of any potential biases from the pooled HRs.
In summary, our updated meta-analysis suggests that PD-L1 expression is not associated with OS or PFS of patients with ovarian cancer. Due to high heterogeneity, the data are not highly reliable. Furthermore, PD-L1 expression was observed to be associated with histological type or FIGO stage of ovarian cancer, specifically with non-Asian patients with this disease. Thus, additional research with prospective large-cohort studies involving diverse population should be conducted to conclusively demonstrate whether PD-L1 expression has a significant prognostic value in ovarian cancer.

\section{Acknowledgments}

This work was supported by a grant from the Seoul National University Hospital Research Fund (Grant No. 03-20190260).

\section{Conflict of interest}

No potential conflict of interest relevant to this article was reported.

\section{Ethical approval}

Ethical approval was not required for our meta-analysis as only previously published studies were analyzed.

\section{References}

1. Torre LA, Bray F, Siegel RL, Ferlay J, Lortet-Tieulent J, Jemal A. Global cancer statistics, 2012. CA Cancer J Clin 2015;65:87-108.

2. Jayson GC, Kohn EC, Kitchener HC, Ledermann JA. Ovarian cancer. Lancet 2014;384:1376-88.

3. Gato-Cañas $M$, Zuazo $M$, Arasanz $H$, Ibañez-Vea $M$, Lorenzo L, Fernandez-Hinojal G, et al. PDL1 signals through conserved sequence motifs to overcome interferon-mediated cytotoxicity. Cell Reports 2017;20:1818-29.

4. Pardoll DM. The blockade of immune checkpoints in cancer immunotherapy. Nat Rev Cancer 2012;12:25264. 


\title{
Obstetrics \& Gynecology Science
}

\author{
Jinlan Piao, et al. Prognostic value of PD-L1
}

5. Hamanishi J, Mandai M, Iwasaki M, Okazaki T, Tanaka Y, Yamaguchi $\mathrm{K}$, et al. Programmed cell death 1 ligand 1 and tumor-infiltrating CD8+ T lymphocytes are prognostic factors of human ovarian cancer. Proc Natl Acad Sci U S A 2007;104:3360-5.

6. Mills A, Peres LC, Abbott S, Meiss A, Schildkraut JM. Characterization of the immune microenvironment of high grade serous ovarian carcinomas in African American women: a study of 112 cases from the African American cancer epidemiology study (AACES). Lab Invest 2018;98:437-8.

7. Huang LJ, Deng XF, Chang F, Wu XL, Wu Y, Diao QZ. Prognostic significance of programmed cell death ligand 1 expression in patients with ovarian carcinoma: a systematic review and meta-analysis. Medicine (Baltimore) 2018;97:e12858.

8. Li M, Li H, Liu F, Bi R, Tu X, Chen L, et al. Characterization of ovarian clear cell carcinoma using target drugbased molecular biomarkers: implications for personalized cancer therapy. J Ovarian Res 2017;10:9.

9. Zhu J, Wen H, Bi R, Wu Y, Wu X. Prognostic value of programmed death-ligand 1 (PD-L1) expression in ovarian clear cell carcinoma. J Gynecol Oncol 2017;28:e77.

10. Webb JR, Milne K, Kroeger DR, Nelson BH. PD-L1 expression is associated with tumor-infiltrating $T$ cells and favorable prognosis in high-grade serous ovarian cancer. Gynecol Oncol 2016;141:293-302.

11. Wang Q, Lou W, Di W, Wu X. Prognostic value of tumor PD-L1 expression combined with CD8+ tumor infiltrating lymphocytes in high grade serous ovarian cancer. Int Immunopharmacol 2017;52:7-14.

12. Mills AM, Peres LC, Meiss A, Ring KL, Modesitt SC, Abbott $\mathrm{SE}$, et al. Targetable immune regulatory molecule expression in high-grade serous ovarian carcinomas in African American Women: a study of PD-L1 and IDO in 112 cases from the African American Cancer Epidemiology Study (AACES). Int J Gynecol Pathol 2019;38:15770.

13. Kim HS, Kim JY, Lee YJ, Kim SH, Lee JY, Nam EJ, et al. Expression of programmed cell death ligand 1 and immune checkpoint markers in residual tumors after neoadjuvant chemotherapy for advanced high-grade serous ovarian cancer. Gynecol Oncol 2018;151:414-21.

14. Darb-Esfahani S, Kunze CA, Kulbe H, Sehouli J, Wienert $S$, Lindner J, et al. Prognostic impact of programmed cell death-1 (PD-1) and PD-ligand 1 (PD-L1) expression in cancer cells and tumor-infiltrating lymphocytes in ovarian high grade serous carcinoma. Oncotarget 2016;7:1486-99.

15. Mesnage SJ, Auguste A, Genestie C, Dunant A, Pain E, Drusch F, et al. Neoadjuvant chemotherapy (NACT) increases immune infiltration and programmed deathligand 1 (PD-L1) expression in epithelial ovarian cancer (EOC). Ann Oncol 2017;28:651-7.

16. Chatterjee J, Dai W, Aziz NH, Teo PY, Wahba J, Phelps $\mathrm{DL}$, et al. Clinical use of programmed cell death-1 and its ligand expression as discriminatory and predictive markers in ovarian cancer. Clin Cancer Res 2017;23:3453-60.

17. Tai H, Yang Q, Wu Z, Sun S, Cao R, Xi Y, et al. PD-L1 expression predicts a distinct prognosis in Krukenberg tumor with corresponding origins. J Immunol Res 2018;2018:9485285.

18. Zou W, Wolchok JD, Chen L. PD-L1 (B7-H1) and PD-1 pathway blockade for cancer therapy: mechanisms, response biomarkers, and combinations. Sci Transl Med 2016;8:328rv4.

19. Sharma P, Allison JP. Immune checkpoint targeting in cancer therapy: toward combination strategies with $\mathrm{Cu}$ rative potential. Cell 2015;161:205-14.

20. Taube JM, Anders RA, Young GD, Xu H, Sharma R, McMiller TL, et al. Colocalization of inflammatory response with B7-h1 expression in human melanocytic lesions supports an adaptive resistance mechanism of immune escape. Sci Transl Med 2012;4:127ra37.

21. Thompson RH, Gillett MD, Cheville JC, Lohse CM, Dong $\mathrm{H}$, Webster WS, et al. Costimulatory B7-H1 in renal cell carcinoma patients: indicator of tumor aggressiveness and potential therapeutic target. Proc Natl Acad Sci U S A 2004;101:17174-9.

22. Chen N, Fang W, Zhan J, Hong S, Tang Y, Kang S, et al. Upregulation of PD-L1 by EGFR activation mediates the immune escape in EGFR-driven NSCLC: implication for optional immune targeted therapy for NSCLC patients with EGFR mutation. J Thorac Oncol 2015;10:910-23.

23. Abiko K, Matsumura N, Hamanishi J, Horikawa N, Murakami $R$, Yamaguchi $K$, et al. IFN- $\gamma$ from lymphocytes induces PD-L1 expression and promotes progression of ovarian cancer. Br J Cancer 2015;112:1501-9.

24. Quandt D, Jasinski-Bergner S, Müller U, Schulze B, Seliger $B$. Synergistic effects of IL-4 and TNFa on the induc- 


\section{Obstetrics \& Gynecology Science}

Vol. 63, No. 3, 2020

tion of B7-H1 in renal cell carcinoma cells inhibiting allogeneic T cell proliferation. J Transl Med 2014;12:151.

25. Krönig H, Kremmler L, Haller B, Englert C, Peschel C, Andreesen $R$, et al. Interferon-induced programmed death-ligand 1 (PD-L1/B7-H1) expression increases on human acute myeloid leukemia blast cells during treatment. Eur J Haematol 2014;92:195-203.

26. Alsaab HO, Sau S, Alzhrani R, Tatiparti K, Bhise K, Kashaw SK, et al. PD-1 and PD-L1 checkpoint signaling inhibition for cancer immunotherapy: mechanism, combinations, and clinical outcome. Front Pharmacol 2017;8:561.

27. Guan J, Lim KS, Mekhail T, Chang CC. Programmed death ligand-1 (PD-L1) expression in the programmed death receptor-1 (PD-1)/PD-L1 blockade: a key player against various cancers. Arch Pathol Lab Med 2017;141:851-61.

28. Cha YJ, Kim HR, Lee CY, Cho BC, Shim HS. Clinicopathological and prognostic significance of programmed cell death ligand- 1 expression in lung adenocarcinoma and its relationship with p53 status. Lung Cancer 2016;97:73-80.

29. Tomioka N, Azuma M, Ikarashi M, Yamamoto M, Sato $\mathrm{M}$, Watanabe $\mathrm{Kl}$, et al. The therapeutic candidate for immune checkpoint inhibitors elucidated by the status of tumor-infiltrating lymphocytes (TILs) and programmed death ligand 1 (PD-L1) expression in triple negative breast cancer (TNBC). Breast Cancer 2018;25:34-42.

30. Jung $H I$, Jeong $D$, Ji S, Ahn TS, Bae SH, Chin S, et al. Overexpression of PD-L1 and PD-L2 is associated with poor prognosis in patients with hepatocellular carcinoma. Cancer Res Treat 2017;49:246-54.

31. Enwere EK, Kornaga EN, Dean M, Koulis TA, Phan T, Kalantarian M, et al. Expression of PD-L1 and presence of CD8-positive T cells in pre-treatment specimens of locally advanced cervical cancer. Mod Pathol 2017;30:57786.

32. Drakes ML, Mehrotra S, Aldulescu M, Potkul RK, Liu Y, Grisoli $A$, et al. Stratification of ovarian tumor pathology by expression of programmed cell death-1 (PD-1) and PD-ligand-1 (PD-L1) in ovarian cancer. J Ovarian Res 2018;11:43.

33. Kleffel S, Posch C, Barthel SR, Mueller H, Schlapbach C,

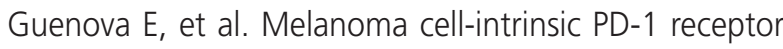
functions promote tumor growth. Cell 2015;162:124256.

34. Mandai M, Hamanishi J, Abiko K, Matsumura N, Baba T, Konishi I. Anti-PD-L1/PD-1 immune therapies in ovarian cancer: basic mechanism and future clinical application. Int J Clin Oncol 2016;21:456-61.

35. Zhu H, Zhang R. Beyond immune suppression: the intrinsic function of PD-L1 in ovarian cancer and melanoma. Transl Cancer Res 2017;6:S102-3.

36. Yoon JY, Stewart JM, Go C, Bernardini M, Clarke B, Shaw $P$, et al. Characterization of the surface expression of PD-1/PD-L1 signaling proteins in high-grade serous ovarian carcinoma. Lab Invest 2017;97:317A-318A.

37. Rimm D, Schalper K, Pusztai L. Unvalidated antibodies and misleading results. Breast Cancer Res Treat 2014;147:457-8. 